\title{
LASERTERAPIA EM ODONTOPEDIATRIA: POSSIBILIDADES TERAPÊUTICAS ${ }^{1}$
}

\author{
Gabriela Rossini Dutra², Nathalia Lucca², Cristiane Medianeira Savian ${ }^{3}$, \\ Cândida Bonadiman Jung ${ }^{4}$, Letícia Westphalen Bento ${ }^{5}$, Simone Pippi \\ Antoniazzi $^{5}$, Maurício Barbieri Mezomo ${ }^{5}$, Bianca Zimmermann dos Santos ${ }^{6}$
}

\section{RESUMO}

O objetivo deste trabalho foi realizar uma revisão narrativa da literatura acerca de como tem se dado o uso da laserterapia em odontopediatria e seus impactos. As buscas foram realizadas nas bases de dados MEDLINE/PubMed e Portal Regional da BVS. Na base de dados MEDLINE/PubMed foram utilizados os descritores MeSH: "lasers", "child", "pediatric dentistry", e na BVS os descritores DeCS: "lasers", "criança", "odontopediatria", em ambas as bases os descritores foram combinados através do operador booleano AND. Foram incluídos artigos publicados entre $2010 \mathrm{e}$ 2020. Visto que nos últimos anos o assunto vem ganhando espaço na Odontologia e tem se destacado por meio de evidências clínicas. O laser representa uma ferramenta adjuvante nos tratamentos, exibindo inúmeras utilidades na manipulação de tecidos moles, tecidos duros e na terapia pulpar. Os resultados encontrados apresentam semelhanças, apesar de observados protocolos variados, entretanto, a maioria dos autores defendem que 0 uso de laser melhora a qualidade do atendimento, bem como, o controle da dor e edema, efeitos do processo inflamatório, promovendo o restabelecimento das funções orais.

Palavras-chave: laser, odontopediatria, terapia a laser, criança.

Eixo Temático: Saúde Materno Infantil (SMI)

\section{INTRODUÇÃO}

O uso do laser ganha o mercado em uma grande escala ascendente, por se tratar de procedimentos na sua grande maioria simples, rápidos e indolores. $\mathrm{Na}$ odontologia, o laser com o passar dos anos, foi inserido com o propósito de minimizar o uso de técnicas invasivas em diferentes casos e a melhorar a qualidade

\footnotetext{
${ }^{1}$ Trabalho desenvolvido durante a disciplina de Estágio Supervisionado III: Atenção à Saúde Bucal Infantojuvenil do curso de Odontologia da UFN.

${ }^{2}$ Acadêmica do Curso de Odontologia - UFN.gabriela.dutra@ufn.edu.br; nathalia.lucca@ufn.edu.br

${ }^{3}$ Mestre em Saúde Materno Infantil - UFN

${ }^{4}$ Mestranda em Saúde Materno Infantil - Universidade Franciscana - UFN

5 Professor(a) do Curso de Odontologia - Universidade Franciscana - UFN

${ }^{6}$ Orientadora. Professora do Curso de Odontologia - Universidade Franciscana - UFN
} 
do atendimento odontopediátrico. Ao contemplar uma estratégia preventiva e terapêutica, a tecnologia corrobora com a ideia da odontologia minimamente invasiva e ainda, mostra-se eficiente e previsível (ONUR, 2020).

Pacientes pediátricos, requerem um cuidado significativo tanto no seu acolhimento, no transoperatório e pós-operatório, visto que os traumas podem se estabelecer a partir da sensação dolorosa e uso de motores que emitem sons e ruídos intermitentes. Por razões como essa, o uso da terapia a laser é bem tolerado por crianças e a cooperação do paciente leva a uma maior satisfação dos pais, dentistas e dos próprios filhos. Procedimentos de caráter invasivo aos protocolos usuais que possibilitem a substituição de instrumentos afiados e geradores de som pelo laser, beneficiam o primeiro contato do paciente com o dentista e o ambiente clínico, garantindo uma boa construção do vínculo e melhor relação entre profissional-paciente em consonância à motivação e estabelecimento de bons hábitos (NAZEMISALMAN; FARSADEGHI; SOKHANSANJ, 2015).

Tecnologia baseada em uma luz emitida de modo uniforme e contínuo sem contato com a área alvo, aplica-se na odontologia em comprimentos de onda que variam na faixa de 193-10600nm e seus respectivos nomes são relacionados aos átomos constituintes no seu interior que estimulados emitem partículas de luz, os fótons. As aplicabilidades do uso do laser na odontologia e estritamente ao campo da odontopediatria atende aos diferentes tipos de tecnologias que cada empresa fornece ao seu produto. Sendo assim, o diferente material constituinte e os diferentes comprimentos de onda, determinam sua aplicação clínica que vai desde a prevenção, detecção e remoção de tecido cariado, procedimentos pulpares, procedimentos em tecidos moles, desinfecção e descontaminação de feridas cirúrgicas, analgesia, redução de inflamação e edema, além de acelerar o processo de cicatrização dos tecidos na cavidade oral (NAZEMISALMAN, FARSADEGHI, SOKHANSANJ, 2015).

Estão descritos na literatura muitos benefícios, sendo que a terapia a laser também apresenta algumas deficiências como o custo elevado, difícil acessibilidade a todos os níveis socioeconômicos e de atenção em saúde bucal, necessidade de 
EDUCAÇÃO, SAÚDE

ETECNOLOGIA

26 A 28 DE OUTUBRO DE 2021

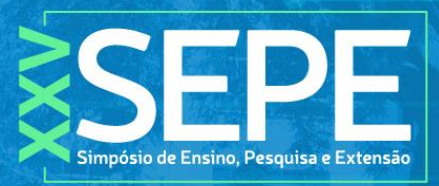

TRABALHO COMPLETO

protocolos de segurança para uso, devido a sua natureza perigosa e ainda, a impossibilidade de uso em todos os campos da odontologia.

Os lasers de baixa intensidade podem ser usados como uma terapia coadjuvante na prática clínica do cirurgião-dentista a favor do bem-estar do paciente ou estratégia complementar a muitos procedimentos terapêuticos, preventivos e restauradores em odontologia, bem como em diversas especialidades como na cirurgia oral, dentística, estomatologia, endodontia, periodontia, etc. Isso significa que a terapia a laser não tem por finalidade substituir protocolos e técnicas que já vêm sendo utilizadas nas condutas clínicas, mas se apresenta como um método complementar na prestação de atendimento odontológico proporcionando inúmeros benefícios. E quando se trata ao atendimento odontológico em crianças esses aspectos são importantes e devem ser levados em consideração, visto que esses sentem dor muitas vezes, tendo comprometimento na qualidade de vida, desconfortos, o que poderia ser evitado ou prevenido com sessões de laserterapia (NAZEMISALMAN; FARSADEGHI; SOKHANSANJ, 2015; OLIVI et al., 2017).

Neste contexto o objetivo deste trabalho é realizar uma revisão de literatura acerca de como tem se dado o uso da laserterapia em odontopediatria e seus impactos.

\section{METODOLOGIA}

O estudo trata de uma revisão narrativa de literatura e para o desenvolvimento desta, foi realizada uma busca de artigos referentes ao tema estudado, nas bases de dados MEDLINE/PubMed e BVS (Biblioteca Virtual em Saúde). A pesquisa foi realizada no mês de julho de 2021. Na base de dados MEDLINE/PubMed foram utilizados os descritores MeSH: "lasers", "child", "pediatric dentistry", e na BVS os descritores DeCS: "lasers", "criança", "odontopediatria", combinados pelo operador booleano AND. Foram incluídos artigos publicados entre 2010 e 2020, devido ao uso do laser ter se tornado mais frequente, neste período.

Foi realizada a leitura dos títulos e resumos de todos os artigos encontrados, para adequada inclusão dos trabalhos relacionados ao tema. Como critério de 
inclusão foi considerado: artigos que contemplassem a temática proposta, publicados em português, inglês e espanhol. O critério de exclusão estabelecido foi artigos que não estivessem com o texto completo disponível.

\section{RESULTADOS E DISCUSSÕES}

\section{Laser em Odontopediatria / Tecidos Moles}

A utilização do laser cirúrgico como forma de tratamento de um caso de Granuloma de células gigantes periféricas decorrente a trauma em um paciente infantil, uma lesão de tecidos moles frequentemente localizada na gengiva, bem como na mucosa alveolar na região posterior da maxila. Visto que tratamentos agressivos são evitados em crianças quando possível considerando o crescimento contínuo, a excisão a laser foi realizada com o objetivo de remover a lesão seguido do exame histopatológico da peça, diante da alternativa os resultados foram clinicamente relevantes e promissores. Os lasers de diodo como este mencionado no relato são ferramentas eficazes para cortes precisos, além disso provocam um selamento de vasos sanguíneos de pequeno calibre evitando sangramentos excessivos e reduzindo complicações no período pós-operatório, agem sobre as fibras nervosas sensoriais diminuindo a capacidade de transmitir estímulos de dor e têm demonstrado efeito bactericida e ação antiinflamatória na cavidade oral (SUYASH; BHATIA, 2018).

O laser tem demonstrado ser uma ótima estratégia complementar para ser aplicada em atendimentos de pacientes pediátricos, os quais rotineiramente apresentam medo, ansiedade e receios frente a sua consulta com o cirurgiãodentista. Há relatos na literatura que demonstram a capacidade da laserterapia para tratar lesões recorrentes de tecidos moles na cavidade oral de maneira indolor e de modo a proporcionar alívio imediato ao paciente. Recentemente foi utilizado um protocolo de uso para terapia a laser de baixa intensidade em um paciente infantil que apresentou queixa de dor e clinicamente úlcera traumática no lábio inferior após anestesia com bloqueio do nervo alveolar inferior, após uma semana do tratamento foi possível verificar melhora significativa com duas aplicações sequenciais do laser, 
além do método provocar analgesia e cicatrização rápida se mostrou uma boa alternativa para lesão de úlcera traumática (CALAZANS et al., 2020).

Para o manejo e tratamento dessas lesões traumáticas, analgésicos e antiinflamatórios são muitas vezes prescritos pelos profissionais com o objetivo de contribuírem para a melhora dos sinais e sintomas dessas alterações em crianças, entretanto uma possibilidade não medicamentosa útil que vem ganhando vez na Odontologia é a aplicação do laser de baixa intensidade para auxiliar no processo cicatricial dos tecidos e mucosa oral e no controle da dor porque aumenta 0 metabolismo, proliferação, maturação, quantidade de tecido de granulação e diminui os mediadores inflamatórios dando início a cicatrização. Neste caso clínico relatado anteriormente o protocolo estabelecido consistiu em duas aplicações sequenciais de infravermelho laser de Diodo, realizadas de modo contínuo em um comprimento de onda de $808 \mathrm{~nm}$, potência de $100 \mathrm{nW}$ e densidade de energia J/cm2 por 5 segundos em torno da extensão de toda úlcera. Em relação aos resultados, após uma semana do tratamento o paciente relatou melhora significativa inclusive para se alimentar e com trinta dias de tratamento a criança apresentou completa cicatrização da região. (CALAZANS et al., 2020).

A frenectomia labial realizada a laser é mais uma das inúmeras utilidades do aparelho abordada em um estudo retrospectivo que menciona a técnica de remoção do freio labial com o auxílio de um laser cirúrgico minimamente invasiva, segura, de fácil execução por um profissional capacitado e previsível. Os autores incluíram um total de 20 frenectomias utilizando o laser em crianças com idade de 8 a 10 anos, sendo o seguinte protocolo seguido: $150 \mathrm{~mJ}, 2,25-3,0 \mathrm{~W}$ e 15-20 pulsos por segundo, com spray de água. Vale lembrar que o acompanhamento foi feito com retornos em 7, 21 e 90 dias após o procedimento e posteriormente em 1, 2, 3 e 4 anos onde todos os pacientes relataram ausência de dor pós-operatória ou desconforto mínimo, nenhum apresentou sangramento ou outras complicações. Um aspecto interessante mencionado na conclusão baseada nos tratamentos e proservação dos casos é a redução considerável do tempo de operação, ou seja, o laser gera otimização do tempo quando comparado a cirurgia de frenectomia com métodos convencionais (OLIVI; GENOVESE; OLIVI, 2018). 
O tratamento de anquiloglossia com laser odontológico também vem sendo abordado em pacientes pediátricos, pois promove um tratamento mais eficiente e confortável para a criança e o dentista em comparação com o bisturi convencional. Ainda na literatura há relatos de procedimentos de expansão rápida da maxila como um dos tratamentos mais comuns de deficiência transversa da maxila a laser de baixa intensidade, sendo uma das alternativas recomendadas para auxiliar no reparo biológico dos tecidos orais e ósseos. O estudo buscou fazer alguns questionamentos como os efeitos do laser em pacientes submetidos a expansão rápida da maxila cirúrgica ou não cirúrgica, em relação a efetividade clínica e cicatrização da ferida. $O$ comprimento de onda aplicado variou de 660 a $830 \mathrm{~nm}$ com uma faixa de saída de 40-100 mW. Além disso, a maior energia exposta foi $420 \mathrm{~J} / \mathrm{cm} 2$ e o mais baixo foi $100 \mathrm{~J} / \mathrm{cm} 2$. Com base nos ensaios clínicos randomizados disponíveis e que foram incluídos nesta revisão sistemática, o laser de baixa intensidade é melhor para ser utilizado na fase inicial da expansão da maxila por oferecer benefícios no aumento da taxa de remodelação óssea (DAVOUDI; AMROLAHI; KHAKI, 2018; RANGEL et al., 2019).

A mucosite oral faz parte de mais um dos campos de aplicação do laser com finalidade terapêutica, é considerada uma das complicações mais frequentes após a quimioterapia, ocorrendo em aproximadamente 52 a $80 \%$ das crianças em tratamento para o câncer. Tendo em vista que, nos dias atuais, o laser tem se destacado nos tratamentos das mais diversas situações, foi sugerido seu uso para reduzir a severidade de quadros de mucosite oral e aliviar os sintomas. E se tratando de pacientes infantis e adolescentes com presença de estomatites estes experimentam dor expressiva, incômodo, dificuldade para se alimentar adequadamente em decorrência ao desconforto ao mastigar e/ou engolir, alteração da superfície absortiva intestinal, e possibilidade de desnutrição e potencial de infecção (CAUWELS; MARTEANS, 2011; HE et al., 2017).

Nesse contexto, um estudo piloto realizado em 2011, investigou a capacidade de alívio da dor e da cicatrização de feridas com a utilização do laser de baixa intensidade em paciente com mucosite oral induzida por quimioterapia em um grupo de oncologia pediátrica. Todas as crianças foram tratadas usando laser de Diodo 
com comprimento de onda de $830 \mathrm{~nm}$ e uma potência de $150 \mathrm{~mW}$. O mesmo protocolo clínico foi repetido a cada 48 horas até que ocorreu a cicatrização a cada lesão. Após 12 meses, os pacientes foram reavaliados e foram registradas informações sobre sequência de tratamento, sessões de tratamento e frequências relacionadas à sensação de dor e conforto. Ao final foi possível verificar que a laserterapia é uma das terapias de tratamento mais recentes e promissoras, tem se mostrado eficaz para reduzir a gravidade, a duração da mucosite e aliviar a dor desencadeada pela lesão (CAUWELS e MARTEANS, 2011; HE et al., 2018).

\section{Laser em Odontopediatria / Tecidos duros}

A cárie é uma doença prevalente em inúmeros países e influenciada pela dieta. O tratamento com flúor é a maneira mais eficaz de prevenir novas lesões em pacientes com alto risco. Em lesões profundas, com necessidade restauradora, 0 meio mais comum de remoção de tecido cariado é através de instrumentos rotatórios que exibem desvantagens tais como: risco de preparação excessiva, remoção de dentina saudável, risco de afetar a polpa por vibrações e o calor da broca. Essas consequências negativas têm sido os principais motivos para a inclusão do laser (SARMADI; HEDMAN; GABRE, 2013).

Se tratando da prevenção da desmineralização do esmalte, sabe-se que a medida que o flúor tópico é descrito como um elemento essencial no tratamento da cárie dentária no processo de desmineralização e na remineralização, o uso de diversos lasers ainda apresentam controvérsias sobre seus efeitos na estrutura dental. A terapia com flúor apresenta limitações como a necessidade da presença constante em cavidade oral e da qualidade de higiene de cada indivíduo e por esses motivos a proposta do uso do laser como forma complementar em crianças com alto risco de cárie, seriam menos dependentes e mais eficientes. Com base na histologia, os tecidos dentários de dentes decíduos e permanentes variam suas composições químicas, morfológicas e características estruturais. A resposta dos dentes decíduos frente a instabilidade no $\mathrm{pH}$ oral, é baixa e o risco de cárie em pacientes pediátricos é aumentado, pois na dentição decídua os tecidos apresentam 
alto grau de porosidade, juntamente com menor densidade mineral (MOGHADAM et al., 2018).

Com o objetivo de avaliar o efeito do laser de Diodo em reduzir a perda da resistência do esmalte dental após processos de ciclagem de $\mathrm{pH}$, em um comprimento de onda de $810 \mathrm{~nm}$, com e sem o uso de flúor, um estudo in vitro usou sessenta coroas de molares decíduos separando em seis grupos, sendo eles: C não recebeu tratamento; F - aplicação de verniz fluoretado; 2L - irradiação do laser de diodo 2 vezes; 4L- 4 vezes irradiação do laser de diodo; F2L - 2 vezes a irradiação do laser sobre verniz fluoretado; F4L - 4 vezes a irradiação a laser sobre verniz fluoretado. Cada grupo foi submetido a métodos de desenvolvimento de lesões artificiais de cárie. Os resultados mostraram que a menor redução da resistência do esmalte foi observada no grupo 4L, F e F4L e a maior no grupo no grupo C, 2L e F2L. A análise dos dados evidenciou eficácia em todos os tratamentos para prevenção do processo de desmineralização, exceto na terapia a laser 2 vezes e ainda, na presença de flúor, não foram observadas diferenças significativas entre os grupos sem e com flúor. O estudo concluiu que o efeito do laser Diodo na estrutura do esmalte dental e a capacidade de reduzir a perda da resistência é controverso, pois os grupos F, F2L e F4L indicam que o efeito do flúor era considerado semelhante à sua combinação com laser, assim sendo, a irradiação do laser não teve efeito sinérgico sobre o verniz fluoretado. Apesar de que tratamentos com laser e flúor reduziram a perda da resistência da desmineralização do esmalte após processos de ciclagem de $\mathrm{pH}$, o uso combinado do laser com flúor não foi mais eficaz que o tratamento apenas com flúor. (MOGHADAM et al., 2018).

\section{Laser em Odontopediatria / Terapia pulpar}

Assim como um método complementar, a terapia com laser de baixa intensidade na polpa vital de dentes decíduos foi abordada em um estudo com sessenta molares decíduos inferiores de crianças na faixa etária de 5 a 9 anos que foram divididas em quatro grupos: Formocresol Diluído (FC), Hidróxido de Cálcio $(\mathrm{CH})$, Terapia a Laser de Baixa Intensidade (LLLT) e Hidróxido de Cálcio precedido pela Terapia a Laser de Baixa Intensidade (LLLT $+\mathrm{CH}$ ) e acompanhadas em um 
protocolo clínico e radiográfico de 6, 12 e 18 meses pós-operatório para avaliar a eficácia do laser InGaAIP (Twin Laser, MMOptics, São Carlos, SP, Brazil) em um comprimento de onda de 660nm. Com base na análise estatística aos 6 meses, a taxa de sucesso radiográfico para o grupo $\mathrm{FC}$ foi de $100 \%, 60 \%$ para o grupo $\mathrm{CH}$, $80 \%$ para o grupo LLLT e 85,7\% para o grupo LLLT $+\mathrm{CH}$. Após um período de 12 meses, a taxa de sucesso radiográfico para o grupo FC foi de $100 \%$, para o grupo $\mathrm{CH} 50 \%$, 80\% no grupo LLLT e 78,5\% para o grupo LLLT $+\mathrm{CH}$. No período de 18 meses, a taxa de sucesso radiográfico no grupo $\mathrm{FC}$ foi de $100 \%$, no grupo $\mathrm{CH}$ de $66,7 \%$, no grupo LLLT foi de $73,3 \%$ e $75 \%$ no grupo LLLT $+\mathrm{CH}$. Nesse período de tempo maior, o grupo FC não apresentou reabsorção interna e em contrapartida o grupo LLLT + CH apresentou pouca reabsorção interna seguida pelos grupos LLLT e $\mathrm{CH}$. Em relação à formação de barreira de tecido duro, foi observado que no grupo LLLT + CH mais seguido do grupo CH (FERNANDES et al., 2015).

Portanto, a análise evidenciou diferença estatisticamente significativa entre os grupos. Durante o tratamento, não houve relato de sintomatologia dolorosa e/ou sinais de infecção clínica associada ao tratamento pulpar, além disso, nenhum dente mostrou sinais de mobilidade, fístula, inchaço ou inflamação. Uso combinado de Hidróxido de Cálcio e laser, demonstrou maior taxas de sucesso clínico e radiográfico melhores quando comparado ao uso de Hidróxido de Cálcio sozinho. $A$ formação de barreira de tecido duro foi mais frequente no grupo LLLT $+\mathrm{CH}$, seguido pelo $\mathrm{CH}$ e a ausência do mesmo foi no grupo $\mathrm{FC}$, demonstra que o uso do Formocresol não foi capaz de induzir a reparação tecidual. Sugere-se por fim, que a terapia com laser de baixa intensidade, pode ser considerada alternativa para terapias pulpares. Conclui-se que, o efeito benéfico evidenciado pelas interações descritas, ainda não são claras devido à falta de estudos e julga-se necessário pesquisas por meio de ensaios clínicos com maiores amostras e períodos de acompanhamento mais longos para reforçar a segurança do protocolo a laser, pois o tratamento de doenças pulpares dependem do estado do tecido, a condição sistêmica, características dos materiais, mecanismo de ação, $\mathrm{pH}$, perfil de saúde oral e nível de toxicidade para entender a resposta da polpa. (FERNANDES et al., 2015). 


\section{QUFN}

\section{CONCLUSÃO}

O laser é um recurso auxiliar com múltiplas indicações e utilidades, considerado um avanço na área odontológica e atua nos mais diversos tratamentos como em casos de estomatites, aftas, herpes labial, redução de sintomas de erupção dos dentes, queilite angular, entre outros. Além disso, a literatura aponta que o tratamento de condições associando ao uso do laser traz resultados promissores quando se trata principalmente do público infantil, por ser uma alternativa indolor pode ser bastante útil em crianças que sofrem de ansiedade e medo durante sua consulta ao dentista. Por fim, ao lançar mão de novas tecnologias, sempre há uma diversidade de protocolos e resultados controversos no meio clínico, reforçando a importância de revisar a literatura sistematicamente.

\section{REFERÊNCIAS}

CALAZANS, T.A. et al. Protocol for Low-level laser therapy in traumatic ulcer after troncular anesthesia: Case report in pediatric dentistry. Journal of Clinical and Experimental Dentistry, v. 12, n. 2, p. 201-203, 2020.

CAUWELS, R.G.E.C.; MARTENS, L.C. Low level laser therapy in oral mucositis: a pilot study. European Archives of Paediatric Dentistry, v. 12, n. 2, p. 118-123, 2011.

DAVOUDI, A.; AMROLAHI, M.; KHAKI, H. Effects of laser therapy on patients who underwent rapid maxillary expansion; a systematic review. Lasers in Medicinal Science, v. 33, n. 6, p. 1387-1395, 2018.

FERNANDES, A.P. et al. Clinical and radiographic outcomes of the use of Low-Level Laser Therapy in vital pulp of primary teeth. International Journal of Paediatric Dentistry, v. 2, n. 25, p. 144-150, 2015. 
HE, M. et al. A systematic review and meta-analysis of the effect of low-level laser therapy (LLLT) on chemotherapy-induced oral mucositis in pediatric and young patients. European Journal of Pediatrics, v. 177, n. 1, p. 7-17, 2018.

HENRIQUE, A.C.G.; CAZAL, C.; CASTRO, J.F.L. Ação da laserterapia no processo de proliferação e diferenciação celular. Revisão da literatura. Revista do Colégio Brasileiro de Cirurgiões, v. 37, n. 4, p. 295-302, 2010.

KHOSRAVIANI, F. et al. Therapeutic effect of laser on pediatric oral soft tissue problems: a systematic literature review. Lasers in Medical Science, v. 34, n. 9, p. 1735-1746, 2019.

MARTENS, L.C. Laser physics and a review of laser applications in dentistry for children. European Archives of Paediatric Dentistry, v. 12, n. 2, p. 61-67, 2011.

MOGHADAM, N. C. Z. et al. Effects of Laser and Fluoride on the Prevention of Enamel Demineralization: An In Vitro Study. Journal of Lasers in Medical Sciences, v. 9, n. 3, p. 177-182, 2018.

NAZEMISALMAN, B; FARSADEGHI, M; SOKHANSANJ, M. Types of Lasers and Their Applications in Pediatric Dentistry. Journal of Lasers in Medical Sciences, v. 6, n. 3, p. 96-101, 2015.

OLIVI, G. et al. Paediatric laser dentistry. Part 2: Hard tissue laser applications. European Journal Of Paediatric Dentistry, v. 18, n. 2, p. 163-166, 2017.

OLIVI, M.; GENOVESE, M.D.; OLIVI, G. Laser labial frenectomy: a simplified and predictable technique. Retrospective clinical study. European Journal Of Paediatric Dentistry, v. 19, n. 1, p. 56-60, 2018.

ONUR, S.G. Evaluation of Pain Perception and Wound Healing After Laser-Assisted Frenectomy in Pediatric Patients: A Retrospective Comparative Study. 
EDUCAÇÃO, SAÚDE

ETECNỎLGIA

26 A 28 DE OUTUBRO DE 2021

\section{QSUFN}

Photobiomodulation, Photomedicine, and Laser Surgery, v. 39, n. 3, p. 1-7, 2020.

RANGEL, A.G. et al. Treatment of ankyloglossia with dental laser in paediatric patients: Scoping review and a case report. European Journal Of Paediatric Dentistry, v. 20, n. 2, p. 155-163, 2019.

SARMADI, R; HEDMAN, E; GABRE, P. Laser in caries treatment - patients' experiences and opinions. International Journal of Dental Hygiene, v. 12, n. 1, p. 67-73, 2014.

SUYASH, I.A.; BHATIA, R. A Case of Painless Excision. International Journal of Clinical Pediatrics, v. 11, n. 2, p. 135-140, 2018. 